\title{
Pacific
}

Journal of

Mathematics

\section{FOUR DODECAHEDRAL SPACES}

\section{PETER LORIMER}

Volume $156 \quad$ No. 2

December 1992 


\section{FOUR DODECAHEDRAL SPACES}

\section{PETER LORIMER}

Four dodecahedral spaces are constructed from the Coxeter groups $[3,3,5]$ and $[5,3,5]$. Two of these are the Poincaré homology sphere and its hyperbolic analogue discovered by Weber and Seifert (1933). Two are spherical manifolds and two are hyperbolic.

The 120-cell is a tessellation of the sphere $S^{3}$ in 4-dimensional space into dodecahedrons and its group of symmetries is the Coxeter group [3, 3, 5] of order 14400. (See [5, 7, 8 pp. 131-134].) This group has a subgroup $G_{1}$, which can be used to construct the Poincaré homology sphere as the orbit space of the action of $G_{1}$ on $S^{3}[8, \mathrm{pp}$. 123-126].

This paper is based on two observations. First, the group [3, 3, 5] is a homomorphic image of the Coxeter group [5, 3, 5], which is the group of symmetries of a tessellation of 3-dimensional hyperbolic space by dodecahedrons. Second, the group [3, 3, 5] has a subgroup $G_{2}$, which is not isomorphic to $G_{1}$, but has those properties which enable the Poincaré homology sphere to be defined from $G_{1}$.

As a consequence, four dodecahedral spaces can be constructed from $[3,3,5]$. One of them is the homology sphere of Poincare and another is a hyperbolic manifold discovered by Weber and Seifert [11].

1. The Coxeter groups $[3,3,5]$ and $[5,3,5]$. The Coxeter group with Dynkin diagram,

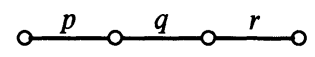

is generated by four elements $a, b, c, d$ subject to the relations

$$
\begin{aligned}
a^{2} & =b^{2}=c^{2}=d^{2}=(a b)^{p}=(b c)^{q}=(c d)^{r} \\
& =(a c)^{2}=(b d)^{2}=(a d)^{2}=1,
\end{aligned}
$$

and is denoted by $[p, q, r]$.

The group $[3,3,5]$ is a central product of two copies of $\operatorname{SL}(2,5)$ extended by an involution which interchanges the two factors. It has order 14400 . 
John Cannon's group theory package CAYLEY was used to show the following.

(I) The group $[3,3,5]$ has nine conjugacy classes of subgroups of index 120 , two of which contain subgroups which intersect $\langle b, c, d\rangle$ trivially. Two subgroups which are representative of these two classes are:

$$
\begin{aligned}
& G_{1}=\langle a d c b d c d a b c a b, a c b d c d b c d c a b, a b c d c d b c d b c b\rangle \\
& G_{2}=\langle a d c b, a c b d c b d c d a b c d a b c\rangle .
\end{aligned}
$$

As the subgroup $\langle b, c, d\rangle$ has index 120 in $[3,3,5]$, it is a complement of each subgroup in each of these classes.

(II) The group $[3,3,5]$ is generated by $a_{1}, b, c, d$ and $a_{2}, b$, $c, d$ where

$$
\begin{aligned}
& a_{1}=a(b c d)^{5} a(b c d)^{5} a, \\
& a_{2}=(b c d)^{5} a_{1}(b c d)^{5} .
\end{aligned}
$$

Also, these quadruples satisfy the relations for $[5,3,5]$, with $a_{1}$ or $a_{2}$ in place of $a$.

It is a consequence of (II) that there are unique homomorphisms,

$$
\varphi, \psi:[5,3,5] \rightarrow[3,3,5],
$$

which satisfy

$$
\begin{array}{llll}
\varphi(a)=a_{1}, & \varphi(b)=b, & \varphi(c)=c, & \varphi(d)=d, \\
\psi(a)=a_{2}, & \psi(b)=b, & \psi(c)=c, & \psi(d)=d .
\end{array}
$$

In these equations and much of the following, the letters $a, b$, $c, d$ represent the generators of both $[3,3,5]$ and $[5,3,5]$, but it should be clear from the context which group is involved.

As $a_{1}$ and $a_{2}$ both lead to the same hyperbolic dodecahedral space, only the first of them will be considered in detail.

The subgroups $\psi^{-1}\left(G_{1}\right)$ and $\varphi^{-1}\left(G_{2}\right)$ have the same properties in $[5,3,5]$ that $G_{1}$ and $G_{2}$ have in $[3,3,5]$; they have index 120 and intersect $\langle b, c, d\rangle$ trivially.

2. The spherical spaces. In 4-dimensional Euclidean space, the points at distance 1 from the origin form a 3-dimensional manifold, the 3-sphere $S^{3}$; it inherits its "spherical" metric from the space in which it lies. In much the same way that the 2-sphere, $S^{2}$, can be tessellated by 12 regular pentagons to form a regular dodecahedron, so $S^{3}$ can be tessellated by 120 regular dodecahedrons to form the 120-cell. This has 600 vertices, 1200 edges and 720 pentagonal faces. 
The group of symmetries of the 120-cell can be described as follows. Let $\alpha, \beta, \gamma, \delta$ be a flag of the 120-cell, i.e. $\delta$ is one of its dodecahedral cells, $\gamma$ is a pentagonal face in the boundary of $\delta, \beta$ is a boundary edge of $\gamma$ and $\alpha$ is an end point of $\beta$. The 120-cell has symmetries $a, b, c, d$ which are reflections in hyperplanes of the 4-dimensional space in which it lies and satisfy:

$$
\begin{aligned}
& a \text { fixes } \alpha, \beta \text { and } \gamma \text { but not } \delta, \\
& b \text { fixes } \alpha, \beta \text { and } \delta \text { but not } \gamma, \\
& c \text { fixes } \alpha, \gamma \text { and } \delta \text { but not } \beta, \\
& d \text { fixes } \beta, \gamma \text { and } \delta \text { but not } \alpha .
\end{aligned}
$$

These reflections are uniquely determined, they generate the full group of symmetries of the 120-cell and they satisfy all the relations for $[3,3,5]$. In fact, these two groups are isomorphic in this interpretation and, for the rest of this section, they will be identified with one another.

In $[3,3,5]$, as a group of symmetries of the 120-cell, the stabilizers of the elements $\alpha, \beta, \gamma, \delta$ are the subgroups $V=\langle a, b, c\rangle, E=$ $\langle a, b, d\rangle, F=\langle a, c, d\rangle$ and $C=\langle b, c, d\rangle$ respectively.

These subgroups have orders $24,12,20$ and 120, respectively, and $[3,3,5]$ acts sharply transitively on the flags of the 120-cell.

Consider the subgroups $G_{1}$ and $G_{2}$ defined in $\S 1$. As each of them is a complement of $C$ in $[3,3,5]$, the interior of $\delta$ is a fundamental region for each of the orbit spaces $S^{3} / G_{1}$ and $S^{3} / G_{2}$, i.e., each point of the interior of $\delta$ lies in a different orbit under the action of $G_{1}$ and $G_{2}$ and each point of $S^{3}$ is in the same orbit as at least one point in the closure of $\delta$ (see $[7,10]$ ). Thus, the orbit spaces can be identified with the closure of $\delta$ with certain identifications among the vertices, edges and faces in its boundary.

The necessary identifications can be calculated using CAYLEY. Those for the subgroups $G_{1}$ give rise to the Poincaré homology sphere, a diagram of which can be found in [8, page 125]. Those for $G_{2}$ are shown in Figure 1 (see next page), at least for the edges and faces.

CAYLEY also shows that $G_{1}$ and $G_{2}$ intersect $V, E$ and $F$ trivially. This shows up in Figure 1 where the vertices are identified in 5 lots of 4 , the edges are identified in 10 lots of 3 and the faces are identified in 6 lots of 2. Consequently, both orbit spaces are spherical manifolds. Moreover, it follows from [1] that $G_{1}$ and $G_{2}$ are the fundamental groups of their orbit spaces. It is well known that $G_{1}$ is isomorphic to $\operatorname{SL}(2,5)$. On the other hand, $G_{2}$ is defined in terms 


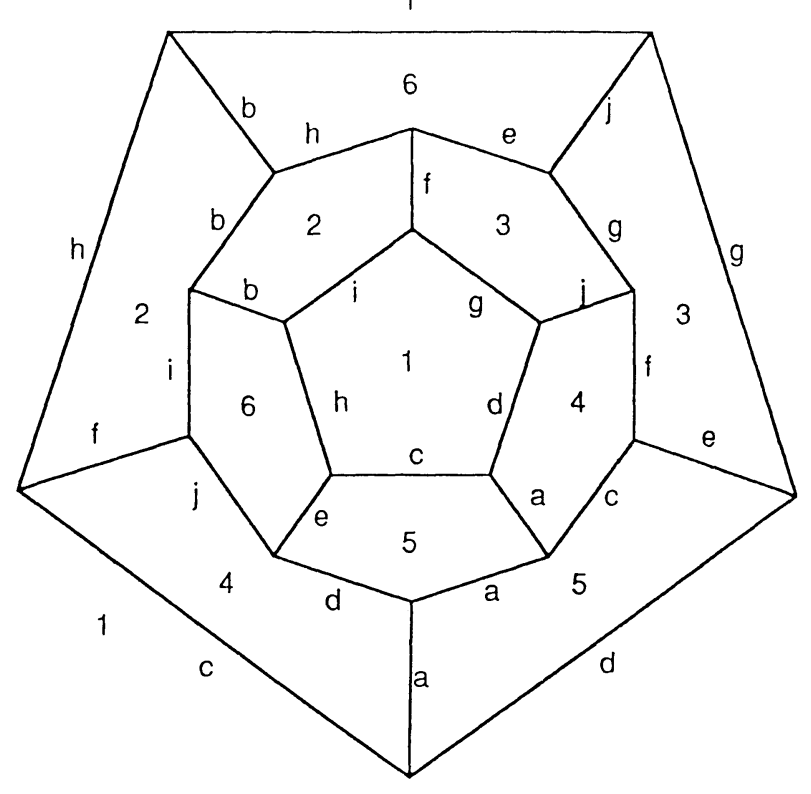

Figure 1

The spherical space

of the given generators, rewritten as $p$ and $q$, by the relations

$$
p^{2}=q^{3}=\left(p q^{2}\right)^{3} \text {. }
$$

This is a solvable group of order 120 in which the derived group has index 15. Thus the first homology group of the corresponding manifold is the cyclic group of order 15 .

3. The hyperbolic spaces. Three-dimensional hyperbolic space, $\mathbb{H}^{3}$, can be tessellated by regular dodecahedrons with 12 meeting at each vertex and 5 meeting at each edge [2]. The group of symmetries of this tessellation can be described in the same way as the group of the 120-cell. A flag $\alpha, \beta, \gamma, \delta$ is defined in the same way and in terms of it, reflections $a, b, c, d$ in planes of $\mathbb{H}^{3}$ are identified, just as in $\S 1$. With $a, b, c, d$ as generators, the group of symmetries of this tessellation of hyperbolic space is $[5,3,5]$ and the stabilizers in $[5,3,5]$ of $\alpha, \beta, \gamma, \delta$ are $V=\langle a, b, c\rangle, E=\langle a, b, d\rangle$, $F=\langle a, c, d\rangle$ and $C=\langle b, c, d\rangle$, respectively, just as before.

As $C$ is a complement of both $\varphi^{-1}\left(G_{1}\right)$ and $\varphi^{-1}\left(G_{2}\right)$ in $[5,3,5]$, the orbit spaces of both these subgroups have the interior of $\delta$ as a fundamental region and the resulting manifold can be identified with the closure of $\delta$, with certain elements of its boundary identified with each other. Again, the identifications are easily calculated using 


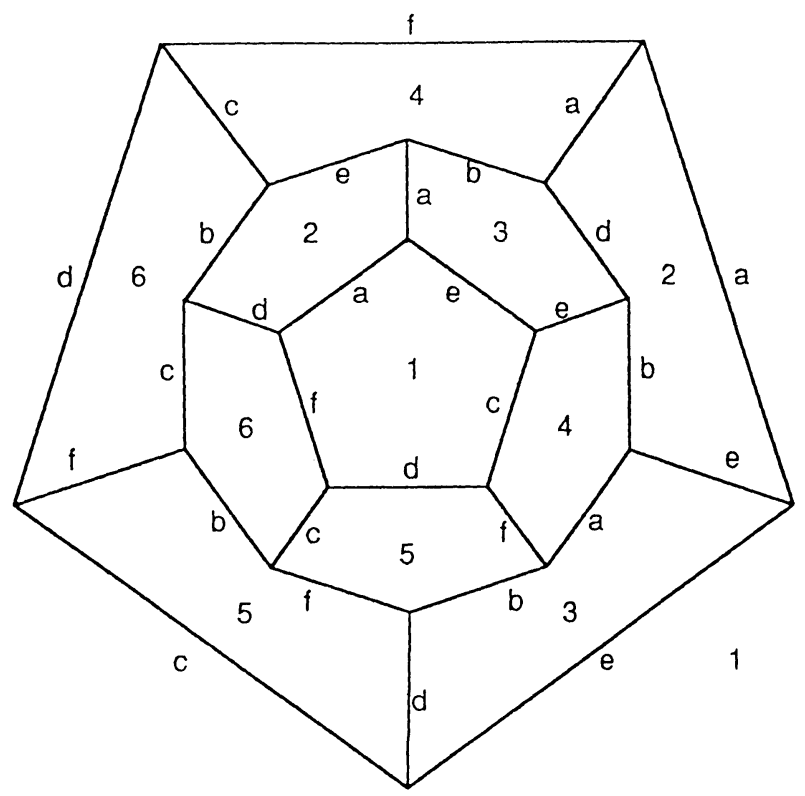

Figure 2

The hyperbolic space

CAYLEY. That arising from $\varphi^{-1}\left(G_{1}\right)$ turns out to be the WeberSeifert manifold $[3, \mathbf{1 1}]$ and the other is illustrated in Figure 2.

By the Theorem in [1], the groups $\varphi^{-1}\left(G_{1}\right)$ and $\varphi^{-1}\left(G_{2}\right)$ are the fundamental groups of these manifolds. The Reidemeister-Schreier process in CAYLEY shows that $\varphi^{-1}\left(G_{1}\right)$ is generated by

$$
\begin{aligned}
p=b a c b d a c d b c d a, & q=a d c b d c b a b c d c, \\
r & =a b a b c d a b c d b a, \quad s=c d c d c b d c b a b a,
\end{aligned}
$$

and has defining relations,

$$
\begin{aligned}
& p q s r p r^{-2} s^{-1} q p s=p q r^{-1} p r^{-2} s^{-1} q s r^{-1} s^{-1} q \\
& \quad=p q^{2} s r s p q s r^{2} p^{-1} s^{-1} q=p q s r^{2} p^{-1} r s^{-1} r^{-1} s^{-1} q^{-1} p^{-1} s^{-1} q=1 .
\end{aligned}
$$

In the representation of $[5,3,5]$ on the left cosets of $\varphi^{-1}\left(G_{1}\right)$, the representation of each of the stabilizers $V, E, F$ and $C$ is faithful and the image of $\varphi^{-1}\left(G_{1}\right)$ intersects their images, and the images of all their conjugates, trivially. Thus, in $[5,3,5]$, the subgroup $\varphi^{-1}\left(G_{1}\right)$ contains no element, except the identity, fixing a point of $\mathbb{H}^{3}$. Thus, by the Theorem in $[1], \varphi^{-1}\left(G_{1}\right)$ is the fundamental group of its orbit space, the Weber-Seifert manifold. From the given relations, it is easy to calculate that the quotient group of $\varphi^{-1}\left(G_{1}\right)$ over its derived group 
is isomorphic to $C_{5} \times C_{5} \times C_{5}$, thus confirming the calculation at the end of [11]. This is the first homology group of the space.

The same is true, in principle, for the other hyperbolic manifold, except that $\varphi^{-1}\left(G_{2}\right)$ is generated by

$$
\begin{aligned}
p & =b a b c b a b c d b a b c a, & q & =d c b a c d c b a b \\
r & =a c b a d c d c d c b a, & & s=d c d a b c,
\end{aligned}
$$

with defining relations

$$
\begin{aligned}
& p r^{2} s p^{-1} r s^{2}=p r^{2} q^{-1} s^{-1} p^{-1} s^{-2} r^{-1} s^{-1} q \\
& \quad=p r^{2} q^{-1} s^{-1} r^{-1} p^{-1} q^{-1} s p^{-1} q^{-1} \\
& \quad=p r^{2} q^{-1} s p s^{-1} r^{-1} p s^{-1} q^{-1} s p s^{-1} r^{-1} q^{-1}=1 .
\end{aligned}
$$

This is the fundamental group of the second hyperbolic manifold: its derived quotient is $C_{15} \times C_{15}$ and that is the first homology group of the space.

As each of $\varphi^{-1}\left(G_{1}\right)$ and $\varphi^{-1}\left(G_{2}\right)$ intersect each of $V, E, F$ trivially, the manifolds both inherit a hyperbolic metric from $\mathbb{H}^{3}$ : they are hyperbolic manifolds.

Everything in this section remains valid with $a_{2}$ in place of $a_{1}$ and $\psi$ in place of $\varphi$. However, the resulting manifolds turn out to be the same.

\section{REFERENCES}

[1] M. A. Armstrong, The fundamental group of the orbit space of a discontinuous group, Proc. Camb. Phil. Soc., 64 (1968), 299-301.

[2] H. S. M. Coxeter, Regular honeycombs in hyperbolic space, Proceedings International Congress of Mathematicians, Amsterdam, III (1954), 154-169.

[3] H. S. M. Coxeter and W. O. Moser, Generators and Relations for Discrete Groups, Springer-Verlag, New York, Fourth Edition.

[4] D. Hilbert and S. Cohn-Vossen, Geometry and the Imagination, Chelsea Publishing Co., New York, 1952.

[5] M. M. Hilden, M. T. Lozano, J. M. Montesinos, and W. C. Whitten, On universal groups and three-manifolds, Invent. Math., 87 (1987), 441-456.

[6] P. Lorimer, Towards a 3-dimensional model of the 120-cell, Math. Intelligencer, 11 (1989), 61.

[7] B. Maskit, On Poincaré's theorem for fundamental polygons, Adv. in Math.? 7 (1971), 219-230.

[8] J. M. Montesinos, Classical Tessellations and Three-Manifolds, Springer-Verlag, Berlin, Heidelberg, 1987.

[9] J. Stillwell, Classical Topology and Combinatorial Group Theory, SpringerVerlag, New York, 1980. 
[10] W. Thurston, The geometry and topology of three-manifolds, Princeton University Press, (to appear).

[11] C. Weber and H. Seifert, Die beiden Dodekaederäume, Math. Z., 37 (1933), 237-253.

Received February 7, 1991. The research reported here was carried out with the help of Research grants from the University of Auckland, the New Zealand Universities Grants Committee and the New Zealand Lottery Grants Board.

UNIVERSITY OF AUCKLAND

P. O. Box 92019

Auckland, New Zealand 



\title{
PACIFIC JOURNAL OF MATHEMATICS \\ Founded by \\ E. F. BeCKenbach (1906-1982) F. Wolf (1904-1989)
}

\section{EDITORS}

\author{
V. S. VARADARAJAN \\ (Managing Editor) \\ University of California \\ Los Angeles, CA 90024-1555 \\ vsv@math.ucla.edu \\ Herbert Clemens \\ University of Utah \\ Salt Lake City, UT 84112 \\ clemens@math.utah.edu \\ F. Michael Christ \\ University of California \\ Los Angeles, CA 90024-1555 \\ christ@math.ucla.edu \\ THOMAS ENRIGHT \\ University of California, San Diego \\ La Jolla, CA 92093 \\ tenright@ucsd.edu
}

\author{
Nicholas ERcolani \\ University of Arizona \\ Tucson, AZ 85721 \\ ercolani@math.arizona.edu \\ R. FINN \\ Stanford University \\ Stanford, CA 94305 \\ finn@gauss.stanford.edu \\ VAughan F. R. Jones \\ University of California \\ Berkeley, CA 94720 \\ vfr@math.berkeley.edu \\ STEVEN KeRCKHOFF \\ Stanford University \\ Stanford, CA 94305 \\ spk@gauss.stanford.edu
}

\author{
C. C. MOORE \\ University of California \\ Berkeley, CA 94720
}

MARTIN SCHARLEMANN

University of California

Santa Barbara, CA 93106

mgscharl@henri.ucsb.edu

\author{
HAROLD STARK \\ University of California, San Diego \\ La Jolla, CA 92093
}

\section{SUPPORTING INSTITUTIONS}

UNIVERSITY OF ARIZONA
UNIVERSITY OF BRITISH COLUMBIA
CALIFORNIA INSTITUTE OF TECHNOLOGY
UNIVERSITY OF CALIFORNIA
MONTANA STATE UNIVERSITY
UNIVERSITY OF NEVADA, RENO
NEW MEXICO STATE UNIVERSITY
OREGON STATE UNIVERSITY

\author{
UNIVERSITY OF OREGON \\ UNIVERSITY OF SOUTHERN CALIFORNIA \\ STANFORD UNIVERSITY \\ UNIVERSITY OF HAWAII \\ UNIVERSITY OF TOKYO \\ UNIVERSITY OF UTAH \\ WASHINGTON STATE UNIVERSITY \\ UNIVERSITY OF WASHINGTON
}

The Supporting Institutions listed above contribute to the cost of publication of this Journal, but they are not owners or publishers and have no responsibility for its content or policies.

\begin{abstract}
Mathematical papers intended for publication in the Pacific Journal of Mathematics should be in typed form or offset-reproduced (not dittoed), double spaced with large margins. Please do not use built up fractions in the text of the manuscript. However, you may use them in the displayed equations. Underline Greek letters in red, German in green, and script in blue. The first paragraph must be capable of being used separately as a synopsis of the entire paper. In particular it should contain no bibliographic references. Please propose a heading for the odd numbered pages of less than 35 characters. Manuscripts, in triplicate, may be sent to any one of the editors. Please classify according to the 1991 Mathematics Subject Classification scheme which can be found in the December index volumes of Mathematical Reviews. Supply name and address of author to whom proofs should be sent. All other communications should be addressed to the managing editor, or Elaine Barth, University of California, Los Angeles, California 90024-1555.

There are page-charges associated with articles appearing in the Pacific Journal of Mathematics. These charges are expected to be paid by the author's University, Government Agency or Company. If the author or authors do not have access to such Institutional support these charges are waived. Single authors will receive 50 free reprints; joint authors will receive a total of 100 free reprints. Additional copies may be obtained at cost in multiples of 50 .
\end{abstract}

The Pacific Journal of Mathematics (ISSN 0030-8730) is published monthly except for July and August. Regular subscription rate: $\$ 190.00$ a year (10 issues). Special rate: $\$ 95.00$ a year to individual members of supporting institutions.

Subscriptions, orders for numbers issued in the last three calendar years, and changes of address should be sent to Pacific Journal of Mathematics, P.O. Box 969, Carmel Valley, CA 93924, U.S.A. Old back numbers obtainable from Kraus Periodicals Co., Route 100, Millwood, NY 10546.

The Pacific Journal of Mathematics at P.O. Box 969, Carmel Valley, CA 93924 (ISSN 0030-8730) is published monthly except for July and August. Second-class postage paid at Carmel Valley, California 93924, and additional mailing offices. Postmaster: send address changes to Pacific Journal of Mathematics, P.O. Box 969, Carmel Valley, CA 93924.

\section{PUBLISHED BY PACIFIC JOURNAL OF MATHEMATICS, A NON-PROFIT CORPORATION} Copyright (C) 1992 by Pacific Journal of Mathematics 


\section{PACIFIC JOURNAL OF MATHEMATICS}

Volume $156 \quad$ No. $2 \quad$ December 1992

Surfaces in the 3-dimensional Lorentz-Minkowski space satisfying 201 $\Delta x=A x+B$

Luis Alías, Angel FerRandez and Pascual LuCAS

Lie algebras of type $D_{4}$ over number fields

209

BRUCE ALLISON

Subsemigroups of completely simple semigroups

251

Anne Antonippillai and Francis PAStiJn

Studying links via closed braids. VI. A nonfiniteness theorem

JOAN Birman and William W. MENASCO

Minimal orbits at infinity in homogeneous spaces of nonpositive curvature

MARÍA J. DRUETTA

Generalized horseshoe maps and inverse limits

SARAH ElizABETH Holte

Determinantal criteria for transversality of morphisms

DAN LAKSOV and ROBERT SPEISER

Four dodecahedral spaces

PETER LORIMER

Semifree actions on spheres

MONICA NiCOLAU

Conformal deformations preserving the Gauss map

ENALDO VERGASTA

Hecke eigenforms and representation numbers of arbitrary rank lattices 371

LYNNE WALLING 